\title{
The Effect of Ketorolac on Postoperative Hemoglobin Level after Vaginal Reconstructive Surgery
}

\author{
Shivani Shah ${ }^{1 *}$, Dana Thompson ${ }^{1}$ and Howard Goldstein ${ }^{2}$ \\ *1Department of Obstetrics and Gynecology, Christiana Care Health System, 4755 Ogletown-Stanton Road, Newark, DE \\ ${ }^{2}$ Department of Female Pelvic Surgery and Reconstructive Surgery, Christiana Care Health System, 4735 Ogletown-Stanton Road, \#1208, Newark, DE
}

*Correspondence to: Shivani Shah, M.D. , Department of Obstetrics and Gynecology, Christiana Care Health System, 4755 Ogletown-Stanton Road, Newark, DE 19718, Phone: (302)733-6565, Fax: (302)733-3340, shshah@christianacare.org

Received: March 26, 2018; Accepted: April 16, 2018; Published: April 26, 2018;

\begin{abstract}
Objectives: Ketorolac is an appealing alternative to narcotics in postoperative pain management, however, there is concern for postoperative bleeding. We sought to evaluate and compare the effect of ketorolac on postoperative bleeding and to assess its impact on postoperative pain control in patients undergoing vaginal reconstructive surgery.

Methods: This is a retrospective cohort study of women who had vaginal reconstructive surgery at Christiana Care Health System between January 1, 2014-December 31, 2014. Data on preoperative and postoperative hemoglobin levels as well as total narcotic use (defined as $1 \mathrm{mg}$ of intravenous hydromorphone) was collected for each patient. Data analysis was performed using a chi- square test and unpaired t-tests. Multiple regression and linear regression analyses were performed to assess the effect of ketorolac use on hemoglobin and total narcotic use.

Results: 129 vaginal surgery cases were identified in the study period. 54 (41.9\%) patients received ketorolac and 75 (58.1\%) patients did not receive ketorolac. There was no statistically significant difference in mean narcotic use between the ketorolac group and the no ketorolac group ( $3.85 \mathrm{mg}$ vs $3.0 \mathrm{mg}, \mathrm{p}=0.194)$. Total ketorolac use was not associated with an increase in estimated blood loss. Among patients who received ketorolac, there was no significant difference in pre-operative and post-operative hemoglobin when adjusting for estimated blood loss.
\end{abstract}

Conclusion: This study found that that there was no difference in change in hemoglobin with the use of ketorolac. The total amount of narcotic use was not decreased in patients given ketorolac postoperatively for additional pain control.

\section{Introduction}

Pain control in the postoperative period has been studied extensively in the gynecological literature. Currently, standard postoperative pain control includes a combination of narcotics and non-steroidal anti-inflammatory drugs (NSAIDs). Narcotic medications have well documented side effects including nausea, vomiting, constipation, dizziness, somnolence and a decreased respiratory drive. NSAIDs such as ketorolac have become an attractive option for pain control as an alternative to opioid medications. The effectiveness of ketorolac on postoperative pain control has been well established [1-3]. There is, however, a concern with the effect of ketorolac use on postoperative bleeding [4].

Ketorolac like other NSAIDs can prolong bleeding time by inhibiting platelet aggregation and thromboxaneA2 production $[4,5]$. Early studies on the hemostatic effects of ketorolac have shown prolongation of bleeding time as well as an increased risk for gastrointestinal bleeding in the postoperative period [6,7]. Nevertheless, there are conflicting reports in the literature. A recent meta-analysis [8] of 27 randomized controlled trials examining postoperative bleeding with ketorolac use did not show a significant increase in bleeding compared to controls. Additionally, the meta analysis also showed superior pain control with ketorolac compared with controls, and equally effective pain control when compared to opiates.

Postoperative bleeding after vaginal reconstructive surgery is a well established complication and can present in one of two ways. First, with vaginal bleeding noted several hours after the procedure that may be caused by bleeding from the vaginal cuff or vascular pedicles. Secondly, with little or no vaginal bleeding but deteriorating vital signs, indicating a possible retroperitoneal hematoma.

\section{Materials and Methods}

This is an IRB approved retrospective cohort study of women who had vaginal reconstructive surgery at Christiana Care Health System between January 1 2014-December 31 2014. Patients were identified through the institutional perioperative database using CPT codes for various vaginal procedures. All patients that underwent a vaginal reconstructive procedure done by a board certified Female Pelvic Medicine and Reconstructive Surgery surgeon were included in the data set. A sequential retrospective chart review of patients who underwent vaginal reconstructive surgeries was then conducted. Data was collected through manual review of electronic and hand written paper charts. Patients who underwent additional laparoscopic procedures were excluded. Additionally, patient with a history of 
chronic narcotic use, history of chronic pain and a history of bleeding disorders were excluded. Inclusion criteria included patients over the age of 18 years who underwent a vaginal reconstructive procedure requiring at least an overnight hospital stay. Patient age, BMI, ethnicity, indication, length of procedure, and length of stay were recorded. Administration of Ketorolac was determined by both the surgeon or the anesthesiologist. Ketorolac use, timing of dosage, and total dosage were recorded. For each patient, the 24 hour and 48-hour postoperative narcotic requirement were recorded. Postoperative complications such as blood transfusions, return to the operating room, ileus, were also recorded.

One of the secondary outcomes was to analyze the use of narcotic medications in those patients that received ketorolac in comparison to those that did not. As a variety of narcotic medications were used depending on surgeon preference, narcotic medications were converted to $1 \mathrm{mg}$ IV hydromorphone using an online opioid conversion calculator [9]. The conversion was based upon standard dosing preparations. The time period of 24 hours began upon admission to the post anesthesia care unit.

Data was analyzed using chi-square test for categorical variables and t-test of continuous variables. Linear regression analyses were used to compare ketorolac use with narcotic usage and hemoglobin levels. The latter analysis was adjusted for estimated blood loss during the surgery.

\section{Results}

There were 176 vaginal reconstructive surgery cases were identified during the study period. Based on the exclusion criteria, 47 cases were excluded. Of the remaining 129 vaginal cases, 54 (41.9\%) patients received ketorolac and $75(58.1 \%)$ patients did not receive ketorolac. The group that received ketorolac were younger (56.0 vs 66.1, $\mathrm{p}=0.003)$. A large proportion of the study population was Caucasian $(\mathrm{n}=113,87.6 \%)$. There was no difference in body mass index in both groups. There was no difference in length of stay between the two groups (Table 1). Estimated blood loss (EBL) during the surgery was significantly different in both groups, with the ketorolac group having a lower volume $(84.5 \mathrm{~mL}$ vs. $117.1 \mathrm{~mL}, \mathrm{p}=0.049)$. The difference in preoperative hemoglobin levels and postoperative hemoglobin levels were not significantly different between the two groups (Table 2). Additionally, there was no significant difference in hemoglobin when comparing the total dose of ketorolac with the decrease in hemoglobin level postoperatively $(\mathrm{p}=0.81)$. One patient required a postoperative blood transfusion. This patient, who did not receive ketorolac, had a hemoglobin $>7$, but had clinical symptoms for acute blood loss anemia.

There was no difference in the mean narcotic use between the two groups (Table 3). The total amount of ketorolac used did not have a significant difference in narcotic usage $(\mathrm{p}=0.75)$. No significant difference was seen in narcotic use on postoperative day (POD) \#1 or POD\#2 in the ketorolac group. However, when adjusted for age, there was a significant decrease in narcotic use in the ketorolac group on POD\#0 $(\mathrm{p}=0.045)$. Nineteen patients did not receive any postoperative analgesia, of which nine belonged to the ketorolac group and ten belonged to the no ketorolac group.
Table 1: Demographics

\begin{tabular}{|l|c|c|c|}
\hline & \multicolumn{2}{|c|}{ Ketorolac Use } & \\
\hline Age (yrs) & no ketorolac & ketorolac & p-value \\
\hline BMI (mean SD) & $66.10(13.3)$ & $56.02(14.3)$ & $<0.001$ \\
\hline Race/Ethnicity (n\%) & $29.9(6.45)$ & $28.69(5.20)$ & 0.30 \\
\hline Caucasian & & & 0.46 \\
\hline African American & $68(90.7)$ & $45(83.3 \%)$ & \\
\hline Hispanic & $3(4 \%)$ & $6(11.1 \%)$ & \\
\hline Other & $2(2.7 \%)$ & $1(1.9 \%)$ & \\
\hline Length of Stay (days) & $1.04(0.20)$ & $1.08(0.27)$ & 0.39 \\
\hline $\begin{array}{l}\text { Estimated Blood Loss } \\
\text { (EBL) (mL) }\end{array}$ & 117.1 & 84.47 & 0.05 \\
\hline
\end{tabular}

Table 2: Ketorolac use and Hemoglobin Level

\begin{tabular}{|c|c|c|c|}
\hline & \multicolumn{3}{|c|}{ Ketorolac use } \\
\hline & no ketorolac & ketorolac & p-value \\
\hline Pre-op Hb & 12.92 & 12.68 & 0.36 \\
\hline Post-op Hb & 10.89 & 10.79 & 0.68 \\
\hline $\begin{array}{c}\text { Difference in Average Pre and } \\
\text { Post-op Hb }\end{array}$ & -2.14 & -1.89 & 0.20 \\
\hline
\end{tabular}

Table 3: Ketorolac and Narcotic use

\begin{tabular}{|c|c|c|c|c|}
\hline & \multicolumn{2}{|c|}{ Ketorolac Use } & \multirow{2}{*}{$\begin{array}{c}\text { Mean } \\
\text { Difference }\end{array}$} & \multirow[t]{2}{*}{ p-value } \\
\hline & $\begin{array}{l}\text { no ketorolac } \\
\quad(n=75)\end{array}$ & $\begin{array}{l}\text { ketorolac } \\
(\mathrm{n}=54)\end{array}$ & & \\
\hline $\begin{array}{l}\text { Any Narcotic Use } \\
(\mathrm{n} \%)\end{array}$ & & & & 0.981 \\
\hline No & $11(14.67)$ & $8(14.81)$ & & \\
\hline Yes & $64(85.33)$ & $46(85.19)$ & & \\
\hline Total Narcotics (mg) & & & & \\
\hline Mean (SD) & $3.00(2.95)$ & $3.85(4.04)$ & -0.846 & 0.194 \\
\hline Median (IQR) & $2.00(0.90,4.60)$ & $\begin{array}{c}2.50(0.85 \\
6.15)\end{array}$ & & \\
\hline $\begin{array}{l}\text { Narcotic Dosage } \\
\text { POD \#0 }(\mathrm{mg})\end{array}$ & & & & \\
\hline Mean (SD) & $1.83(1.80)$ & $2.17(2.25)$ & -0.347 & 0.3505 \\
\hline Median (IQR) & $1.20(0.30,2.90)$ & $\begin{array}{c}1.25(0.50 \\
3.50)\end{array}$ & & \\
\hline $\begin{array}{l}\text { Narcotic Dosage } \\
\text { POD \#1 (mg) }\end{array}$ & & & & \\
\hline Mean (SD) & $1.18(1.41)$ & $1.68(2.27)$ & -0.499 & 0.157 \\
\hline Median (IQR) & $0.80(0.00,1.60)$ & $\begin{array}{l}0.85(0.00 \\
2.45)\end{array}$ & & \\
\hline
\end{tabular}


In order to account for a potential confounder of increased pain leading to the addition of ketorolac to the regimen, a subanalysis comparing narcotic use in patients who received ketorolac on POD\#0 was conducted. Patients who did not receive ketorolac on POD\#0 were removed from the analysis. There was no difference in narcotic use in this group of patients $(\mathrm{p}=0.70)$

\section{Discussion}

Our findings show that there was no difference in hemoglobin level postoperatively between the ketorolac and no ketorolac group. Additionally, there was no difference in narcotic use between the two groups. There was no difference in narcotic use on POD\#0 or POD\#1. When adjusting for age, however, there was a significant decrease in narcotic use on POD\#0 ( $\mathrm{p}=0.045)$.

The current literature on whether there is a significant increase in bleeding with ketorolac is conflicting [10-13]. Strom et al (1996) reported a $50 \%$ increase in bleeding time in healthy subjects four hours after administration of Ketorolac [10]. They also showed that there was a $10 \%$ postoperative hemorrhage rate compared to $2 \%$ in patient who only received opioids. Another study showed an increase in bleeding time by 1 minute and 46 seconds in patients that were treated with IM Ketorolac [7]. Conrad et al. showed that bleeding time increased from 4.9 minutes to 7.8 minutes in patients who received IM ketorolac four times a day for five days. An important distinction to make between these studies is the use of bleeding time as the indicator of abnormal bleeding. Bleeding time has been shown to be a reliable marker of abnormal bleeding in patients who receive antiplatelet agents [7]. However, it is unclear if bleeding time could predict clinically significant compromise in hemostasis in the postoperative period [14]. Importantly, there was no association noted between dosage of ketorolac and an increase in bleeding $(\mathrm{p}=0.1692)$. This is a different finding from the literature where there was dose dependent relationship with an increase in bleeding [14].

The effect of ketorolac on pain is well established. Studies in both surgery and gynecology literature have shown a morphine sparing effect with the use of ketorolac in the postoperative period. In this study, unadjusted analyses showed no difference in narcotic usage based on if patient received ketorolac. There was also no dose dependent decrease noted in opiate usage noted. Interestingly, the mean narcotic use was slightly higher in the group that received ketorolac (3.86mg vs. $3.52 \mathrm{mg}$ ), but not significant. A reason that could account for this finding is that ketorolac was given postoperatively to patients who had higher narcotic requirements. When this was accounted for, by eliminating all patients who did not receive ketorolac on POD\#0 from the ketorolac group, the mean narcotic use was lower in the ketorolac group. This finding was not significant. However, the sample size became significantly smaller $(n=38)$ when these patients were removed from analysis. Adjusted analysis showed a significant decrease in narcotic use on POD\#0, but not on POD\#1. Additionally, there was no difference shown when comparing the total dosage of ketorolac with total narcotic use. These findings are not consistent with the literature which has shown a decrease in narcotic use with the use of ketorolac postoperatively.
The narcotic use overall was noted to be low in both groups. A study by Crisp et al. (2017) [15] comparing PCA to nurse administered analgesia postoperative in patients who underwent vaginal reconstructive surgery noted similar dosage of IV hydromorphone, with total hydromorphone use as $3.56 \mathrm{mg}$, and $1.803 \mathrm{mg}$ and $1.770 \mathrm{mg}$ on POD\#0 and POD\#1 respectively. Narcotic usage postoperative after abdominal hysterectomies are significantly higher as noted in a study by Moon et al (2011) [16] with a 24 hour consumption of $4.2 \mathrm{mg}$ $\pm 2.3 \mathrm{mg}$. An alternative hypothesis is that these surgeries may not be painful enough to show a true difference in narcotic consumption, and may require a larger study group to show a difference.

A strength of this study is that this question as not been answered in current literature specifically in Urogynecology. We also attempted to adjust for any confounders with linear regression analyses. All surgeries were preformed by board certified FPMRS surgeons at this institution who provide a consistent level of care. The limitations of the study include that it is a retrospective analysis. Additionally, the sample size is relatively small, and is potentially not large enough to show a true difference between hemoglobin change. Bleeding was assessed through a comparison of preoperative and postoperative hemoglobin. As ketorolac is a prostaglandin inhibitor, its effects are primarily on platelet function and a better parameter to study may be coagulation studies. These parameters were not available in our retrospective analysis but should be included in any prospective studies examining this question. Future directions should include a comparison of ketorolac with IV Tylenol on narcotic use.

\section{References}

1. Brown CR, Moodie JE, Wild VM, Bynum LJ (1990) Comparison of intravenous ketorolac tromethamine and morphine sulfate in the treatment of postoperative pain. Pharmacotherapy 10: 116S-121S. [crossref]

2. Parker RK, Holtmann B, Smith J, White PF (1994) Use of ketorolac after lower abdominal surgery: effect on analgesic requirement and surgical outcome. Anesthesiology 80:6-12.

3. Sevarino FB, Sinatra RS, Paige D, et al. (1992) The efficacy of intramuscular ketorolac in combination with intravenous PCA morphine for postoperative pain relief. J Clin Anesth 4:285-8.

4. Buckley MM, Brogden RN (1990) Ketorolac: A review of its pharmacodynamic and pharmacokinetic properties, and therapeutic potential. Drugs 39:86-109.

5. Greer IA (1990) Effects of ketorolac tromethamine on hemostasis. Pharmacotherapy 10:71S-76S.

6. Rogers JE, Fleming BG, Macintosh KC, et al. (1995) Effect of timing of ketorolac administration on patient-controlled opioid use. Br J Anaesth 75:15-8.

7. Singer AJ, Mynster CJ, McMahon BJ (2003) The effect of IM ketorolac tromethamine on bleeding time: A prospective, interventional, controlled study. Am J Emerg Med 21:441-443

8. Gobble RM, Hoang HL, Kachniarz B, et al. (2014) Ketorolac does not increase perioperative bleeding: a meta-analysis of randomized controlled trials. Plast Reconstr Surg 133:741-55.

9. McCauley, David (2017) Opioid Analgesic Converter

10. Strom BL, Berlin JA, Kinman JL, et al. (1996) Parenteral ketorolac and risk of gastrointestinal and operative site bleeding: A postmarketing surveillance study. JAMA 275: 376-382.

11. Gallagher J, Blauth J, Fornadley JA (1995) Perioperative Ketorolac tromethamine and postoperative hemorrhage in cases of tonsillectomy and adenoidectomy. Laryngoscope 105: 606-609

12. Gobble RM, Hoang HL, Kachniarz B, et al. (2014) Ketorolac does not increase perioperative bleeding: a meta-analysis of randomized controlled trials. Plast Reconstr Surg 133:741-55.

13. Conrad KA, Fagan TC, Mackie MJ, et al. (1988) Effects of ketorolac tromethamine on hemostasis in volunteers. Clin Pharmacol Ther 43:542-546.

14. Lind SE (1991) The bleeding time does not predict surgical bleeding. Blood 77 $2547-2552$ 
Shivani Shah (2018) The Effect of Ketorolac on Postoperative Hemoglobin Level after Vaginal Reconstructive Surgery

15. Moon YE, Lee YK, Lee J, Moon DE (2011) The effects of preoperative intravenous acetaminophen in patients undergoing abdominal hysterectomy. Arch Gynecol Obstet 284:1455-60
16. Crisp CC, Bandi, S; Kleeman, SD, et al. (2012) Patient-controlled versus scheduled, nurse-administered analgesia following vaginalreconstructive surgery: a randomized trial. Am J Obstet Gynecol 207:433.e1-6

\section{Citation:}

Shivani Shah, Dana Thompson, Howard Goldstein (2018) The Effect of Ketorolac on Postoperative Hemoglobin Level after Vaginal Reconstructive Surgery. Integr Gyn Obstet $J$ Volume 2(2): 1-4 